\title{
The hidden consequences of the changing cardiac surgical population
}

\author{
Angela Jerath, BSc, MD, MSc, FRCPC, FANZCA · Duminda N. Wijeysundera, MD, PhD, FRCPC
}

Received: 29 March 2018/Accepted: 4 April 2018/Published online: 31 May 2018

(C) Canadian Anesthesiologists' Society 2018

The characteristics of adults presenting for cardiac surgery have changed dramatically over the last 20 years. With rising life expectancy, these individuals are now older, frailer, and burdened with chronic illness. ${ }^{1,2}$ Furthermore, advancements in interventional cardiology and percutaneous interventions have meant that open cardiac surgical procedures increasingly consist of higher-risk procedures, such as multi-valve or aortic replacement procedures. ${ }^{1,2}$ Although improvements in perioperative care have kept the overall mortality risk low (1-3\%) among cardiac surgical patients, a subgroup still experiences complicated protracted postoperative recoveries that are associated with longer hospitalizations and elevated risks of short- and long-term mortality. In this issue of the Journal, McIsaac et al. present a populationbased cohort study of adult cardiac surgical patients that provides important new data to inform our understanding of this problem. ${ }^{3}$

The authors assembled a cohort of 111,740 patients who underwent five cardiac surgical procedures in Ontario between 2002 and 2013. Based on the postoperative length of stay in the intensive care unit (ICU), duration of critical

A. Jerath, BSc, MD, MSc, FRCPC, FANZCA .

D. N. Wijeysundera, MD, PhD, FRCPC ( $\square)$

Department of Anesthesia and Pain Management, Toronto

General Hospital, Toronto, Canada

e-mail: d.wijeysundera@utoronto.ca

A. Jerath, BSc, MD, MSc, FRCPC, FANZCA .

D. N. Wijeysundera, MD, PhD, FRCPC

Department of Anesthesia, University of Toronto, Toronto, Canada

D. N. Wijeysundera, MD, PhD, FRCPC

Li Ka Shing Knowledge Institute, St. Michael's Hospital, 30

Bond Street, Toronto, ON M5B 1W8, Canada illness was classified as short ( $\leq$ two days), moderate (three to nine days), or prolonged ( $\geq$ ten days). The investigators then performed a risk-adjusted analysis to determine the association of ICU length of stay with oneyear mortality and healthcare resource utilization (i.e., costs, discharge to an institutional facility, hospital readmissions). Perhaps unsurprisingly, patients who experienced moderate or prolonged durations of ICU stay were generally older and sicker. For example, patients with prolonged ICU stays ( $\geq$ ten days) were more likely to be elderly $(>70 \mathrm{yr}$ ) individuals who underwent emergent complex cardiac surgical procedures. They also had a greater burden of chronic illness and a higher number of preoperative ICU admissions.

More importantly, this relatively small subgroup experienced disproportionately high risks of long-term mortality and consumed considerably more healthcare resources. For example, while patients with prolonged ICU stays comprised only $3 \%$ of the entire cohort, they occupied over $30 \%$ of total ICU bed days and experienced almost $30 \%$ of all deaths in the year after surgery. Compared with patients with short ( $\leq$ two days) ICU stays, this high-risk subgroup experienced much higher adjusted risks of one-year mortality (hazard ratio 8.7), discharge to a destination other than home (odds ratio 7.5), and hospital readmission (odds ratio 1.3).

As the authors themselves state, these findings do not prove a causal relationship between prolonged ICU stay and subsequent outcomes. Specifically, residual confounding is highly likely in this population-based study, given that the data sources did not capture several important and relevant perioperative factors (e.g., baseline functional status, left ventricular function, severity of illness, vasopressor support, mechanical cardiac support, massive bleeding, post-surgical complications). Thus, the 
poor outcomes of patients with prolonged ICU stay were, in part, explained by this subgroup disproportionately including acutely ill emergency surgical patients with limited time to optimize medical comorbidities, patients who required pre-surgical life-saving cardiorespiratory support, and patients with additional complications (e.g., acute kidney injury, sepsis). In addition, while using postoperative length of ICU stay as a surrogate measure of postoperative critical illness is a simple and reasonable approach, length of ICU stay is plausibly affected by hospital-level factors, such as institutional practice with respect to standard duration of postoperative ICU stay and the frequency of ICU discharge delays secondary to lack of available ward beds. Nonetheless, these limitations do not negate the major contribution of this study by McIsaac et al., which is to describe the epidemiology of cardiac surgical patients with prolonged critical illness with respect to their characteristics, outcomes, and consumption of healthcare resources. There is no doubt that clinicians, administrators, and policy-makers must pay attention to this small but very high-risk subgroup of patients.

How do these study findings compare with the current literature? The results reported by McIsaac et al. are largely consistent with the expanding body of literature describing adverse outcomes in frail elderly patients who are exposed to acute physiologically stressful illness or major surgical interventions. ${ }^{4}$ The non-surgical critical care literature has closely examined the relative contributions of patients' baseline health status and acute critical illness on the risks of morbidity and mortality among ICU survivors. ${ }^{5}$ Growing evidence has shown that pre-hospitalization patient risk factors significantly influence long-term survival, whereas the severity and type of critical illness have greater influence on short-term survival. ${ }^{4}$ In the perioperative literature, observational studies focused on cardiac surgical patients have shown the impact of comorbidities (e.g., heart failure, chronic obstructive pulmonary disease, renal insufficiency, atrial fibrillation), emergency surgery, inotropic drug support, complex surgical procedures, and prolonged cardiopulmonary bypass on the morbid trajectory for individual patients. ${ }^{6}$ Prior studies have also documented the increase in mortality and resource utilization among elderly cardiac surgical patients with longer ICU stays secondary to both cardiac complications (low cardiac output states, pulmonary hypertension, arrhythmias) and noncardiac complications (respiratory failure, dialysis, sepsis). ${ }^{1,7}$

This present study by McIsaac et al. raises important follow-up questions, namely how best to identify this subgroup of high-risk cardiac surgical patients and what wider implications these findings have for patients and healthcare systems. Accurate preoperative identification of these patients would be highly beneficial for perioperative teams. For example, such risk stratification would enable more careful clinical decision-making for surgery. Improved decision-making would entail greater consideration of the extent and duration of the planned surgical procedure, consideration of alternative minimally invasive techniques, and serious consideration of the merits of non-operative management of the underlying cardiovascular condition. In addition, early identification of these patients would allow for a more informed discussion with patients and care-givers regarding their expectations, risks of prolonged hospitalization, and the impact on long-term outcomes (e.g., survival, disability, cognitive function, need for long-term care). Nevertheless, identifying these patients accurately is not simple. Currently used mortality risk prediction tools for cardiac surgical patients, such as the EuroScore and Cardiac Anesthesia Risk Evaluation score, are predominantly limited to predicting short-term mortality. ${ }^{8,9}$ A validated predictive index for long-term mortality has been developed for patients undergoing coronary artery bypass graft surgery, ${ }^{10}$ but further research is required to provide more appropriate risk tools specifically designed for highrisk elderly patients undergoing complex cardiac surgical procedures. Such tools should incorporate preoperative prognostic information likely more relevant to older patients, such as markers of frailty, physical function, and mental function. Given that elderly patients with lifethreatening comorbidities place greater weight on the risk of disability than the risk of death when selecting various healthcare interventions, ${ }^{11}$ new risk prediction tools should also consider other important outcomes, such as serious postoperative functional and cognitive decline.

These improved tools should help facilitate the highly individualized and patient-centred process of weighing the potential benefit of a complex cardiac surgical procedure against the risks of postoperative death, prolonged complex in-hospital recovery, and significant new long-term disability. Overall long-term survival of elderly patients undergoing cardiac surgery shows similar patterns to ageand sex-matched population cohorts, but survival is considerably lower than in younger patients. $^{12}$ The worsened survival relative to younger patients is consistent with the effects of comorbid diseases, agerelated physiologic decline, and age-related functional decline on perioperative risk. Conversely, the similarity in survival to age- and sex-matched controls does suggest that overall case selection for cardiac surgery is reasonable. Especially with older surgical patients, it is also important to consider outcomes beyond survival alone, such as postoperative quality of life. ${ }^{11}$ Prior research has shown that many elderly surgical patients do experience an improvement in health-related quality of life, but there is a smaller undefined subgroup (9-18\%) who instead 
experience a decline in quality of life in association with poor physical function, vitality scores, and regret about their decision to undergo surgery. ${ }^{12,13}$ Thus, there is a need for good preoperative decision-making tools to aid discussion among patients, families, and healthcare teams. These tools will also facilitate a shared preoperative decision-making process that aims to align treatment outcomes with patients' values and goals of care.

The number of cardiac surgical patients requiring longer ICU and hospital stays will continue to rise with the aging population in most economically advanced countries. ${ }^{14}$ These trends raise several challenges for healthcare teams and policy makers. More patients requiring longer hospital stays will increase pressure on the limited supply of acute care hospital beds. Prolonged ICU stays will limit the number of cardiac surgeries that can be performed, which in turn will impact surgical waiting lists and potentially raise the urgency of surgery for some patients. Especially in hospitals where a finite number of ICU beds must meet the acute care demands of both medical and surgical services, longer ICU admissions will impede timely access to ICU care for patients who are acutely ill because of medical causes and noncardiac surgical procedures. ${ }^{15}$ Critical care is also an expensive resource, with the costs of care being substantially higher for cardiac surgical ICU beds (day 1 US $\$ 5,166$, day 2-30 US $\$ 2,060-2,325$ ) than general surgical and medical ICU beds (day 1 US $\$ 2,002-$ 2,636, day 2-30 US\$1,733-2,084). ${ }^{16}$ Survivors of prolonged ICU care will also subsequently incur additional healthcare costs after their ICU stay related to their longer post-ICU ward care, need for rehabilitation in continuing care facilities, and possibly need for discharge to long-term institutional care. These issues, which are not unique to cardiac surgery patients, raise important healthcare policy questions regarding how to facilitate (and fund) sufficient expansion of post-acute care facilities to support continued access to acute care beds.

Thus, this population-based study by McIsaac et al. sets the stage for an important line of investigation for perioperative medicine. The substantial healthcare impact of this relatively small subgroup of high-risk cardiac surgical patients is clear. Moving forward, we need better approaches to accurately identify patients who will incur prolonged ICU stay. Such tools will improve informed consent, facilitate shared decision-making for surgery, inform discussion surrounding patients' wishes regarding prolonged care, and allow better planning of resources needed to manage these high-risk patients. Additionally, we need to develop approaches for mitigating the risks of deleterious outcomes associated with prolonged postoperative critical illness. Potential options include early optimization of modifiable risk factors, developing specialized care pathways for these high-risk patients, and planning for appropriate resource allocation for this highrisk subgroup. Anesthesiologists are at the forefront of witnessing this changing tide in the surgical patient population. We play a key expanding role in perioperative medicine, which places our specialty in an ideal position to develop new solutions for improving the care of our most complex and vulnerable patients.

\section{Les conséquences cachées de l'évolution de la population chirurgicale cardiaque}

Les caractéristiques démographiques des adultes candidats à la chirurgie cardiaque ont considérablement changé au cours des 20 dernières années. Avec l'allongement de l'espérance de vie, ces individus sont maintenant plus âgés, plus fragiles et aux prises avec des maladies chroniques. ${ }^{1,2}$ En outre, les progrès de la cardiologie interventionnelle et des interventions percutanées signifient que les interventions chirurgicales à cœur ouvert sont de plus en plus des procédures à haut risque, telles que des procédures multivalvulaires ou de remplacement de l'aorte ascendante. $^{1,2}$ Même si l'amélioration des soins périopératoires a maintenu le risque de mortalité globale bas (de $1 \%$ à $3 \%$ ) parmi les patients chirurgicaux cardiaques, un sous-groupe de patients présente toujours des convalescences postopératoires compliquées et prolongées qui sont associées à des durées d'hospitalisation plus longues et à des risques accrus de mortalité à court et long terme. Dans ce numéro du Journal, McIsaac et coll. présentent une étude de cohorte basée sur une population de patients chirurgicaux cardiaques adultes procurant d'importantes données nouvelles pour notre compréhension de ce problème. ${ }^{3}$

Les auteurs ont assemblé une cohorte de 111740 patients qui ont bénéficié de cinq procédures chirurgicales cardiaques en Ontario entre 2002 et 2013. La durée de leur état critique a été classée en fonction de leur durée de séjour en unité de soins critiques (USC) : courte ( $\leq 2$ jours), moyenne ( 3 à 9 jours $)$ ou prolongée ( $\geq 10$ jours). Les investigateurs ont alors effectué une analyse ajustée pour le risque visant à déterminer l'association existant entre la durée de séjour en USC et la mortalité à un an et l'utilisation des ressources de services de santé (c'est-à-dire, les coûts, le congé vers un établissement d'hébergement, les réhospitalisations). Peut-être sans surprise, les patients ayant connu des durées de séjour en USC moyennes ou prolongées étaient en général plus âgés et plus gravement malades. Par exemple, les patients ayant une durée de séjour en USC $\geq$ 
dix jours étaient plus susceptibles d'être des patients âgés (>70 ans) ayant subi des procédures chirurgicales cardiaques complexes et urgentes. Ils présentaient également la plus grande morbidité de maladie chronique et un plus grand nombre d'admissions préopératoires en USC.

Par-dessus tout, ce sous-groupe relativement petit présentait des risques disproportionnellement élevés de mortalité à long terme et consommait considérablement plus de ressources de santé. Par exemple, tandis que les patients aux séjours prolongés en USC ne représentaient que $3 \%$ de l'ensemble de la cohorte, ils ont occupé plus de $30 \%$ des journées totales d'hospitalisation en USC et ont représenté presque $30 \%$ de tous les décès dans l'année suivant l'intervention. Comparativement aux patients restés à l'USC pendant une courte période ( $\leq$ deux jours), ce sous-groupe à haut risque a présenté des risques ajustés de mortalité à un an (rapport de risque 8,7 ), de congé vers une autre destination que le domicile (rapport de cotes 7,5), et de réadmissions à l'hôpital (rapport de cotes 1,3) beaucoup plus élevés.

Comme l'indiquent les auteurs eux-mêmes, ces constatations ne sont pas la preuve d'un lien de causalité entre le séjour prolongé en USC et l'évolution subséquente. De façon spécifique, un facteur confondant résiduel existe très probablement dans cette étude de population, considérant que les sources des données n'ont pas retenu plusieurs facteurs périopératoires importants et pertinents (à savoir, le statut fonctionnel initial, la fonction ventriculaire gauche, la sévérité de la maladie, le soutien hémodynamique par vasopresseur, le soutien mécanique du cœur, l'hémorragie massive et les complications postopératoires). Ainsi, l'évolution défavorable des patients ayant eu des durées de séjour prolongées en USC était en partie expliquée de manière disproportionnée dans ce sous-groupe par, notamment, les patients gravement malades présentant une urgence chirurgicale et n'offrant qu'un temps limité pour optimiser les comorbidités médicales, les patients nécessitant un soutien cardiorespiratoire préchirurgical vital et les patients ayant des complications supplémentaires (par exemple, insuffisance rénale aiguë, sepsis). De plus, bien que l'utilisation de la durée de séjour postopératoire en USC comme mesure de substitution de la gravité de l'état postopératoire soit une démarche pleine de sens, on peut raisonnablement penser que la durée de séjour en USC est affectée par des facteurs tenant au niveau de l'hôpital, tels que les pratiques de l'établissement concernant la durée standard de séjour postopératoire en USC et la fréquence des retards de congé de l'USC par manque de lits disponibles à l'étage. Néanmoins, ces limites ne remettent pas en cause la principale contribution de cette étude par McIsaac et coll. qui est de décrire l'épidémiologie des patients chirurgicaux cardiaques atteints d'affections vitales prolongées pour ce qui concerne leurs caractéristiques, évolutions et consommations de ressources de soins de santé. Les cliniciens, administrateurs et décideurs politiques vont devoir sans aucun doute prêter une attention particulière à ce sous-groupe de patients, de faible taille, mais à risque très élevé.

Comment situer les constatations de cette étude par rapport aux publications actuelles? Les résultats publiés par McIsaac et coll. s'inscrivent largement dans le corpus croissant de publications décrivant l'évolution délétère des patients âgés et fragiles qui se trouvent exposés à une maladie aiguë physiologiquement stressante ou à des interventions chirurgicales majeures. ${ }^{4}$ La littérature portant sur les soins critiques non chirurgicaux a étudié de manière approfondie les contributions relatives de l'état de santé initial des patients et de la maladie aiguë sur les risques de morbidité et mortalité parmi les survivants en USC. ${ }^{5}$ Des données probantes de plus en plus nombreuses ont montré que les facteurs de risque des patients avant leur hospitalisation ont une influence sur la survie à long terme, tandis que la sévérité et le type de maladie critique ont une plus grande influence sur la survie à court-terme. ${ }^{4}$ Dans la littérature concernant la période périopératoire, des études observationnelles qui se sont intéressées aux patients chirurgicaux cardiaques ont montré les répercussions des comorbidités (par exemple, insuffisance cardiaque, maladie pulmonaire obstructive chronique, insuffisance rénale, fibrillation auriculaire), de la chirurgie pratiquée en urgence, du soutien par inotropes, des procédures chirurgicales complexes et de la durée prolongée de la circulation extracorporelle sur l'évolution morbide des patients. ${ }^{6}$ Des études plus anciennes avaient également documenté une augmentation de la mortalité et du recours aux ressources chez les patients chirurgicaux cardiaques plus âgés et restés plus longtemps en USC à la suite de complications à la fois cardiaques (faible débit cardiaque, hypertension pulmonaire, troubles du rythme) et non cardiaques (insuffisance respiratoire, dialyse, sepsis). ${ }^{1,7}$

L'étude actuelle de McIsaac et coll. soulève d'importantes questions, notamment comment mieux identifier ce sous-groupe de patients chirurgicaux cardiaques à risque élevé et quelles sont plus largement les implications de ces constatations pour les patients et le système de soins de santé. L'identification préopératoire précise de ces patients serait un grand avantage pour les équipes de soins périopératoires. Par exemple, une telle stratification du risque permettrait des prises de décisions cliniques plus prudentes pour la chirurgie. Une amélioration de la décision exposerait à une plus grande prise en compte de l'étendue et de la durée de la procédure chirurgicale envisagée, de la recherche d'autres techniques 
moins invasives et d'une sérieuse analyse des avantages et inconvénients d'une gestion non chirurgicale de la maladie cardiovasculaire sous-jacente. De plus, une identification précoce de ces patients permettrait une discussion plus pertinente avec les patients et les aidants à propos de leurs attentes, des risques d'une hospitalisation prolongée et des répercussions des résultats à long terme (par exemple, survie, handicap, fonction cognitive, nécessité de soins de longue durée). Il n'est cependant pas simple d'identifier ces patients avec exactitude. Les outils actuellement utilisés pour la prédiction du risque de mortalité des patients chirurgicaux cardiaques, tels que l'EuroSCORE et le score d'évaluation du risque en anesthésie cardiaque, sont essentiellement limités à la prédiction de la mortalité à court-terme. ${ }^{8,9}$ Un indice prédictif validé pour la mortalité à long terme a été développé pour les patients subissant une chirurgie de pontage aorto-coronarien, ${ }^{10}$ mais des recherches supplémentaires sont nécessaires pour fournir des outils d'évaluation des risques plus adaptés et conçus spécifiquement pour les patients âgés à risque élevé subissant des procédures chirurgicales cardiaques complexes. Ces outils devraient incorporer de l'information pronostique préopératoire probablement plus pertinente pour les patients plus âgés, tels que des marqueurs de fragilité, de fonction physique et de fonctionnement mental. Compte tenu du fait que les patients âgés atteints de comorbidités mettant leur vie en danger donnent un plus grand poids au risque d'invalidité qu'au risque de décès quand ils doivent choisir entre plusieurs types de soins, ${ }^{11}$ les nouveaux outils de prédiction $\mathrm{du}$ risque doivent aussi envisager d'autres points d'aboutissement importants, comme un important déclin fonctionnel ou cognitif postopératoire.

Ces outils améliorés devraient contribuer à faciliter les processus très individualisés et axés sur le patient visant à établir un équilibre entre l'avantage potentiel d'une procédure chirurgicale cardiaque complexe et les risques de décès postopératoire, de convalescence au cours d'une hospitalisation complexe et prolongée, enfin d'une nouvelle incapacité à long terme significative. La survie globale à long terme des patients âgés subissant une chirurgie cardiaque montre des aspects semblables à celle de cohortes de populations appariées pour l'âge et le sexe, mais la survie est considérablement plus courte que pour des patients plus jeunes. ${ }^{12} \mathrm{La}$ moins bonne survie, par rapport à des patients plus jeunes, correspond aux effets des comorbidités, du déclin physiologique lié à l'âge et au déclin fonctionnel lié à l'âge sur le risque périopératoire. Au contraire, la similitude de la survie par rapport à des témoins appariés pour l'âge et le sexe ne suggère pas que la sélection des cas soit globalement raisonnable pour la chirurgie cardiaque. Avec les patients chirurgicaux plus âgés, il est aussi important d'envisager les conséquences au-delà de la seule survie, notamment la qualité de vie postopératoire. ${ }^{11}$ Des études antérieures ont montré que de nombreux patients chirurgicaux âgés bénéficient effectivement d'une amélioration de leur qualité de vie liée à la santé, mais il existe un petit sousgroupe mal défini (9\% à $18 \%$ ) qui présente, en revanche, une baisse de la qualité de vie en association avec des fonctions physiques et des scores de vitalité médiocres, ainsi que des regrets concernant leur décision de subir l'intervention. ${ }^{12,13}$ Il faut donc disposer de bons outils de prise de décision préopératoire pour contribuer à la discussion entre les patients, les familles et les équipes de soins de santé. Ces outils faciliteront aussi un processus partagé de prise de décision préopératoire visant à aligner les résultats thérapeutiques escomptés avec les valeurs et objectifs de soins des patients.

Le nombre de patients chirurgicaux cardiaques nécessitant des séjours prolongés en USC et à l'hôpital va continuer à croître avec le vieillissement de la population dans la majorité des pays économiquement avancés. ${ }^{14}$ Ces tendances soulèvent plusieurs défis pour les équipes de soins de santé et les décideurs politiques. Davantage de patients nécessitant des séjours hospitaliers plus longs augmenteront la pression sur les capacités limitées en lits de soins aigus. Des séjours plus prolongés en USC limiteront le nombre de chirurgies cardiaques qu'il est possible de pratiquer, ce qui aura naturellement des répercussions sur les listes d'attente chirurgicales et augmentera potentiellement l'urgence de la chirurgie pour certains patients. Ce sera notamment le cas dans les hôpitaux ne disposant que d'un nombre limité de lits en USC et devant répondre aux demandes des services de médecine et de chirurgie : les séjours plus longs en USC empêcheront un accès en temps opportun à des soins en USC pour les patients présentant une maladie aiguë de nature médicale ou à la suite de procédures chirurgicales non cardiaques. ${ }^{15}$ Les soins critiques constituent aussi une ressource dispendieuse, car le coût des soins est substantiellement plus élevé pour les lits d'USC chirurgicale cardiaque que pour les lits en USC chirurgicale ou médicale : respectivement, 5166 US\$ le premier jour, 2060 à 2325 US\$ pour les jours 2 à 30 en USC cardiaque contre 2002 à 2636 US\$ pour le premier jour et 1733 à 2084 US\$ pour les jours 2 à 30 en USC non cardiaque. ${ }^{16}$ Les survivants de séjours prolongés en USC vont ensuite entraîner des dépenses de soins de santé supplémentaires après leur séjour en USC en raison de la suite de leurs épisodes de soins à l'étage, au besoin de réadaptation dans des établissements de soins de continuité et, possiblement un congé dans un établissement de soins de longue durée. Ces problèmes qui ne sont pas propres aux patients chirurgicaux cardiaques soulèvent d'importantes questions de politique de santé sur comment faciliter (et financer) une expansion suffisante des établissements de 
soins de suite à la phase aiguë pour soutenir un accès permanent aux lits de soins aigus.

Ainsi, cette étude de population de McIsaac et coll. jette les bases d'une importante voie de recherche en médecine périopératoire. L'impact substantiel des soins de santé pour ce relativement petit sous-groupe de patients chirurgicaux cardiaques à risque élevé est clair. Pour aller de l'avant, nous avons besoin de disposer de meilleures guides pour identifier correctement les patients qui s'exposeront à des séjours prolongés en USC. Ces outils amélioreront le consentement éclairé, faciliteront la prise de décision partagée pour la chirurgie, enrichiront la discussion autour des souhaits des patients concernant des soins prolongés et permettront une meilleure planification des ressources pour la prise en charge de ces patients à risque élevé. De plus, nous devons développer des démarches de réduction des risques d'évolution délétères associés aux états critiques postopératoires prolongés. Les possibles options incluent une optimisation précoce des facteurs de risque modifiables, le développement de parcours de soins spécialisés pour ces patients à risque élevé et la planification de l'attribution des ressources adaptées à ce sous-groupe à risque. Les anesthésiologistes sont en première ligne pour témoigner de cette nouvelle vague de la population de patients chirurgicaux. Nous jouons un rôle clé dans l'expansion de la médecine périopératoire, ce qui place notre spécialité dans une position idéale pour l'élaboration de nouvelles solutions pour l'amélioration des soins de nos patients les plus difficiles et les plus vulnérables.

Conflicts of interest None declared.

Editorial responsibility This submission was handled by Dr. Philip. M. Jones, Associate Editor, Canadian Journal of Anesthesia.

Funding Duminda N. Wijeysundera is supported in part by a New Investigator Award from the Canadian Institutes of Health Research and a Merit Award from the Department of Anesthesia at the University of Toronto.

\section{Conflits d'intérêts Aucun déclaré.}

Responsabilité éditoriale Cet article a été traité par le $\mathrm{D}^{\mathrm{r}}$ Philip. M. Jones, rédacteur adjoint, Journal canadien d'anesthésie.

Financement Duminda N. Wijeysundera bénéficie du soutien partiel des Bourses salariales pour nouveaux chercheurs des Instituts de recherche en santé du Canada et un Prix de mérite du département d'anesthésie de l'université de Toronto.

\section{References}

1. Buth KJ, Gainer RA, Legare JF, Hirsch GM. The changing face of cardiac surgery: practice patterns and outcomes 2001-2010. Can J Cardiol 2014; 30: 224-30.

2. Pierri MD, Capestro F, Zingaro $C$, Torracca $L$. The changing face of cardiac surgery patients: an insight into a Mediterranean region. Eur J Cardiothorac Surg 2010; 38: 407-13.

3. McIsaac DI, McDonald B, Wong CA, van Walraven C. Longterm survival and resource use in critically ill cardiac surgical patients: a population based study. Can J Anesth 2018; 65. DOI: https://doi.org/10.1007/s12630-018-1159-2.

4. Garland A, Olafson K, Ramsey CD, Yogendran M, Fransoo R. Distinct determinants of long-term and short-term survival in critical illness. Intensive Care Med 2014; 40: 1097-105.

5. Cuthbertson BH, Wunsch $H$. Long-term outcomes after critical illness. The best predictor of the future is the past. Am J Respir Crit Care Med 2016; 194: 132-4.

6. Almashrafi A, Elmontsri M, Aylin P. Systematic review of factors influencing length of stay in ICU after adult cardiac surgery. BMC Health Serv Res 2016; 16: 318.

7. Castillo JG, Silvay G, Chikwe J. Cardiac anesthesia and surgery in geriatric patients: epidemiology, current surgical outcomes, and future directions. HSR Proc Intensive Care Cardiovasc Anesth 2009; 1: 6-19.

8. Dupuis JY, Wang F, Nathan H, Lam M, Grimes $S$, Bourke M. The cardiac anesthesia risk evaluation score: a clinically useful predictor of mortality and morbidity after cardiac surgery. Anesthesiology 2001; 94: 194-204.

9. Roques F, Nashef SA, Michel P, et al. Risk factors and outcome in European cardiac surgery: analysis of the EuroSCORE multinational database of 19030 patients. Eur J Cardiothorac Surg 1999; 15: 816-22; discussion 822-3.

10. Wu C, Camacho FT, Wechsler AS, et al. Risk score for predicting long-term mortality after coronary artery bypass graft surgery. Circulation 2012; 125: 2423-30.

11. Fried TR, Bradley EH, Towle VR, Allore H. Understanding the treatment preferences of seriously ill patients. N Engl J Med 2002; 346: 1061-6.

12. Gjeilo KH, Wahba A, Klepstad P, Lydersen S, Stenseth $R$. Survival and quality of life in an elderly cardiac surgery population: 5-year follow-up. Eur J Cardiothorac Surg 2013; 44: e182-8.

13. Abah $U$, Dunne $M, \operatorname{Cook} A$, et al. Does quality of life improve in octogenarians following cardiac surgery? A systematic review. BMJ Open 2015; 5: e006904.

14. Etzioni DA, Liu JH, O'Connell JB, Maggard MA, Ko CY. Elderly patients in surgical workloads: a population-based analysis. Am Surg 2003; 69: 961-5.

15. Harris $S$, Singer $M$, Rowan $K$, Sanderson $C$. Delay to admission to critical care and mortality among deteriorating ward patients in UK hospitals: a multicentre, prospective, observational cohort study. Lancet 2015; 385(Suppl 1): S40.

16. Gershengorn HB, Garland A, Gong MN. Patterns of daily costs differ for medical and surgical intensive care unit patients. Ann Am Thorac Soc 2015; 12: 1831-6. 